\title{
INHALT DES SIEBENUNDSIEBZIGSTEN BANDES
}

\section{ABTEILUNG}

O. LAMPSIDES, Collectanea Planudea und die Chronike Synopsis von Konstantinos Manasses . . I

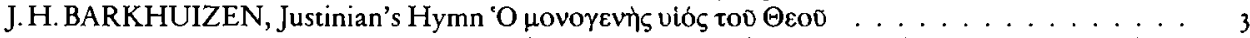
H. MIYAKAWA/A.KOLLAUTZ, Ein Dokument zum Fernhandel zwischen Byzanz und China

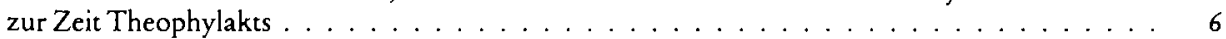

A. KARPOZELOS, Realia in Byzantine Epistolography X-XII c. . . . . . . . . . . . . . 20

V. PUCKO, Das Apsisfresko der Karmravor-Kirche in Aštarak (Armenien) . . . . . . . . . . . . 38

B. BALDWIN, The Authorship of the Timarion . . . . . . . . . . . . . . . . . . 233

MARIAN HARTMANN SHOTWELL, The Question of a Thoman Recension of Aeschylus . . . 238

G. L. HUXLEY, Textual Topics in the Chronicle of Eusebios . . . . . . . . . . . . . . . . . . 257

J. MOORHEAD, Theoderic, Zeno and Odovaker . . . . . . . . . . . . . . . . . . . . . 260

ALICE-MARY M.TALBOT, Old Age in Byzantium . . . . . . . . . . . . . . . . 267

\section{ABTEILUNG}

I Calendari in metro innografico di Cristoforo Mitileneo, a cura di ENRICA FOLLIERI, I. II. Besprochen von $\mathrm{CH}$. HANNICK

Byzanz - wieder ein Weltreich. Das Zeitalter der Makedonischen Dynastie. Teil I . . Nach dem Geschichtswerk des Johannes Skylitzes. Übers., eingel. u. erklärt von H.THURN. Besprochen von J.-L. VAN DIETEN

H.HUNGER, Anonyme Metaphrase zu Anna Komnene, Alexias XI-XIII. Besprochen von

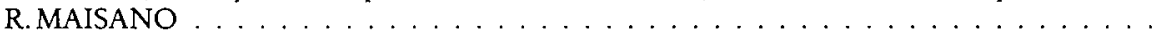

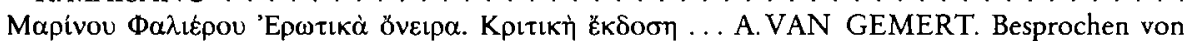

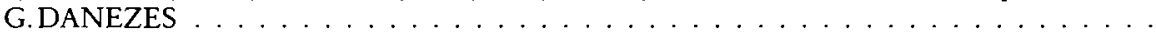

G.SPADARO, Studi di filologia cretese. Besprochen von G.DANEZES . . . . . . . . . . .

GUDRUN ENGBERG, Prophetologium. Pars altera: Lectiones anni immobilis. Fasc. I. II. Bespro-

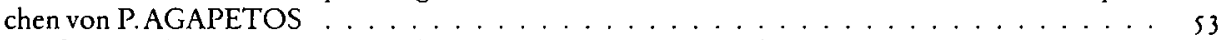

Cyril of Alexandria, Select Letters. Ed. L. R. WICKHAM. Besprochen von PAULINE ALLEN . . . 54

J.H.DECLERCK, Maximi Confessoris Quaestiones et Dubia. Besprochen von R. RIEDINGER . ss

Anonymus in Ecclesiasten Commentarius qui dicitur Catena Trium Patrum. Ed. S. LUCÀ. Besprochen von A.CERESA-GASTALDO

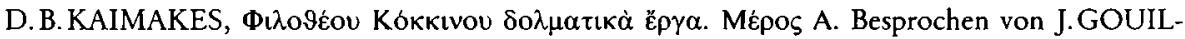

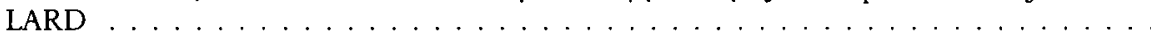

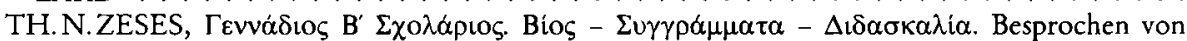
G.PODSKALSKY

GABRIELE WINKLER, Das Armenische Initiationsrituale. Besprochen von S. BROCK . . . . . .

V.BEŠEVIIEV, Die protobulgarische Periode der bulgarischen Geschichte. Besprochen von G.PRINZING

K. L. NOETHLICHS, Beamtentum und Dienstvergehen. Zur Staatsverwaltung in der Spätantike. Besprochen von M.CLAUSS

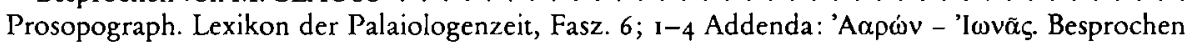
von D.M.NICOL

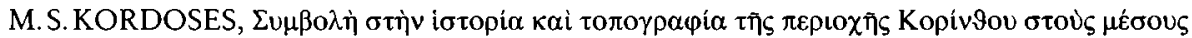
Xpóvous. Besprochen von F. HILD . . . . . . . . . . . . . . . . . .

E. COCHE DE LA FERTÉ, L'Art de Byzance. Besprochen von Z. KÁDÁR . . . . . . . . . . . . . .

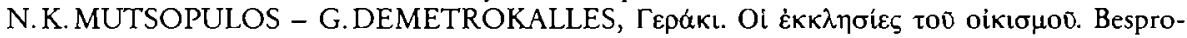
chen von U.PESCHLOW

KARIN M.SKAWRAN, The Development of Middle Byzantine Fresco Painting in Greece. Besprochen von D.I. PALLAS

K. WEITZMANN, GAIANE ALIBEGAŠVILI, ANELI VOLSKAJA u.a., Die Ikonen. Besprochen von D.I.PALLAS

M.-M.GAUTHIER, Straßen des Glaubens. Besprochen von V. H. ELBERN 
PH. MALINGOUDIS, Die mittelalterlichen kyrillischen Inschriften der Hämus-Halbinsel. Teil I. Besprochen von I.DUJČEV . . . . . . . . . . . . . . . . . . . . . . .

W.SELB, Orientalisches Kirchenrecht, I. Die Geschichte des Kirchenrechts der Nestorianer . . . Besprochen von S. TROIANOS . . . . . . . . . . . . . . . . . .

W.HÜBNER, Zodiacus Christianus. Besprochen von P. KUNITZSCH . . . . . . . . . . . . .

Zenobii Athoi proverbia vulgari ceteraque memoria aucta edidit ...W. BÜHLER. IV 2, nr. I-40. Besprochen von L.G. WESTERINK . . . . . . . . . . . . . . . .

J.KARAYANNOPULOS / G. WEISS, Quellenkunde zur Geschichte von Byzanz (324-1453). Besprochen von F. WINKELMANN . . . . . . . . . . . . . . . . . . 280

G.A. KENNEDY, Greek Rhetorik under Christian Emperors. Besprochen von R. MAISANO . . . 283

Scholia in Aristophanem. ... II, fasc. I Scholia vetera et recentiora in Aristophanis Vespas edidit W.J. W. KOSTER. Besprochen von A. KLEINLOGEL . . . . . . . . . . . . . . 28

LIA RAFFAELLA CRESCI, Malco di Filadelfia, Frammenti. Besprochen von B. BALDWIN . . . . 290

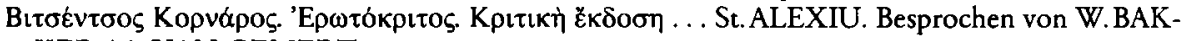
KER / A.VAN GEMERT . . . . . . . . . . . . . . . . . .

Das Register des Patriarchats von Konstantinopel. I. Teil ... Hrsg. von H. HUNGER und O. KRESTEN ... - Studien zum Patriarchatsregister von Konstantinopel. I. Hrsg. von H.HUNGER ... - Das Register des Patriarchats von Konstantinopel. Indices. Erstellt von CAROLINA CUPANE. Besprochen von C. MANGO . . . . . . . . . . . . . . . . . . . . . . . . 297

B. LAVAGNINI, Alle origini del verso politico. Besprochen von J.KODER . . . . . . . . . . . . . . $\quad 298$

The Hagiopolites. A Byzantine Treatise ... Preliminary edition by J. RAASTED. Besprochen von P.AGAPETOS

M.AUBINEAU, Le traité inédit de christologie de Sévérien de Gabala in Centurionem . . . Besprochen von A.CERESA-GASTALDO . . . . . . . . . . . . . . . . . . . . . 300

B.FLUSIN, Miracle et Histoire dans l'Oeuvre de Cyrille de Scythopolis. Besprochen von L.RYDÉN . . . . . . . . . . . . . . . . . . . . . .

L.KRETZENBACHER, Griechische Reiterheilige als Gefangenenretter. Besprochen von TH.RAFF . . . . . . . . . . . . . . . . . . . . . . 302

G.PASSARELLI, L' Eucologio Cryptense Г. B. VII (sec. X.). Besprochen von S. JANERAS . . . . 304

Corippe (Flavius Cresconius Corippus) ... Texte établi ... par S.ANTĖS. Besprochen von J.BLÄNSDORF

C.CAHEN, Orient et Occident au temps des Croisades. Besprochenvon E.D.HEHL

C.

A. BERGER, Das Bad in der byzantinischen Zeit. Besprochen von A. KARPOZELOS . . . . . . 308

L. HEISER, Das Glaubenszeugnis der armenischen Kirche. Besprochen von F. HEYER . . . . . . . 309

G.H.KRAMER, Ambrosius van Milaan en de geschiedenis. Besprochen von J.-L.VAN DIETEN . 309

Tabula Imperii Byzantini ... Bd. 3: Nikopolis und Kephallenia, von P. SOUSTAL ... Besprochen von D. JACOBY

K.GALLAS / K.WESSEL / M.BORBOUDAKIS, Byzantinisches Kreta. Besprochen von L.KRETZENBACHER . . . . . . . . . . . . . . . . . . . 31

CH. BOURAS, Nea Moni on Chios . . Besprochen von U. PESCHLOW . . . . . . . . . . 3I 3

CHR. WALTER, Art and Ritual of the Byzantine Church. Besprochen von G. GALAVARIS . . . . 315

G.STANZL, Längsbau und Zentralbau als Grundthemen der frühchristlichen Architektur. Besprochen von TH.ULBERT .

Z.ŚWIECHOWSKI / A. RIZZI / R. HAMANN-MAC LEAN, Romanische Reliefs von venezianischen Fassaden. Besprochen von MARA BONFIOLI . . . . . . . . . . . . . . . . .

OLGA GRATZIOU, Die dekorierten Handschriften des Schreibers Matthaios von Myra . . Besprochen von Z.KÁDÁR

RENATE PILLINGER, Studien zu römischen Zwischengoldgläsern ... Besprochen von U.SÜSSENBACH . . . . . . . . . . . . . . . . . . . . . .

D. FEISSEL, Recueil des inscriptions chrétiennes de Macédoine du III ${ }^{e}$ au VI ${ }^{e}$ siècle. Besprochen von KONSTANTINA MENTZU-MEIMARE

R.VOLK, Gesundheitswesen und Wohltätigkeit im Spiegel der byzantinischen Klostertypika. Besprochen von G.E.PENTOGALOS

\section{ABTEILUNG}

Bibliographische Notizen und Mitteilungen 